\title{
Opportunistic Relay Selection in Multicast Relay Networks using Compressive Sensing
}

\author{
Khalil Elkhalil ${ }^{1}$, Mohammed E. Eltayeb ${ }^{2}$, Hussain Shibli ${ }^{1}$, Hamid Reza Bahrami ${ }^{2}$, and Tareq Y. Al-Naffouri ${ }^{1,3}$ \\ ${ }^{1}$ Electrical Engineering Department, King Abdullah University of Science and Technology, Thuwal, Saudi Arabia. \\ ${ }^{3}$ Electrical Engineering Department, King Fahd University of Petroleum and Minerals, Dhahran, Saudi Arabia. \\ Email(s): khalil.elkhalil, hussain.shibli, tareq.alnaffouri@kaust.edu.sa \\ ${ }^{2}$ Department of Electrical and Computer Engineering, The University of Akron, Ohio, USA. \\ Email(s): me33,hrb@uakron.edu
}

\begin{abstract}
Relay selection is a simple technique that achieves spatial diversity in cooperative relay networks. However, for relay selection algorithms to make a selection decision, channel state information (CSI) from all cooperating relays is usually required at a central node. This requirement poses two important challenges. Firstly, CSI acquisition generates a great deal of feedback overhead (air-time) that could result in significant transmission delays. Secondly, the fed back channel information is usually corrupted by additive noise. This could lead to transmission outages if the central node selects the set of cooperating relays based on inaccurate feedback information. In this paper, we introduce a limited feedback relay selection algorithm for a multicast relay network. The proposed algorithm exploits the theory of compressive sensing to first obtain the identity of the "strong" relays with limited feedback. Following that, the CSI of the selected relays is estimated using linear minimum mean square error estimation. To minimize the effect of noise on the fed back CSI, we introduce a back-off strategy that optimally backs-off on the noisy estimated CSI. For a fixed group size, we provide closed form expressions for the scaling law of the maximum equivalent SNR for both Decode and Forward (DF) and Amplify and Forward (AF) cases. Numerical results show that the proposed algorithm drastically reduces the feedback air-time and achieves a rate close to that obtained by selection algorithms with dedicated error-free feedback channels.
\end{abstract}

Keywords-Relay selection, Multicast, Feedback, Decode and Forward, Amplify and Forward, Compressive Sensing.

\section{INTRODUCTION}

The increasing demand for mobile and live streaming applications has triggered great interest in relay-aided multicast networks [1]-[7]. In relay-aided multicast networks, a relay forwards the source's packets to a pool of users interested in the same content. Relaying techniques can be classified, based on their forwarding strategy and required processing at the relay terminals, as decode and forward (DF) or amplify and forward (AF) [5]-[7]. In DF relaying, the relay decodes the source packet, prior to re-encoding and transmitting it to the destination, whereas in the AF relaying, the relay amplifies and retransmits the received packet without decoding it. Occasionally, a relay may have poor channel conditions to either the source or the users or both, therefore, the source has to employ selective relaying techniques that take advantage of the nature of the fading channel and preserve the diversity gains of relay networks [8]. In selective relaying, the source selects a single relay, among multiple relays, with a relatively good channel condition to forward its packets to a group of users.

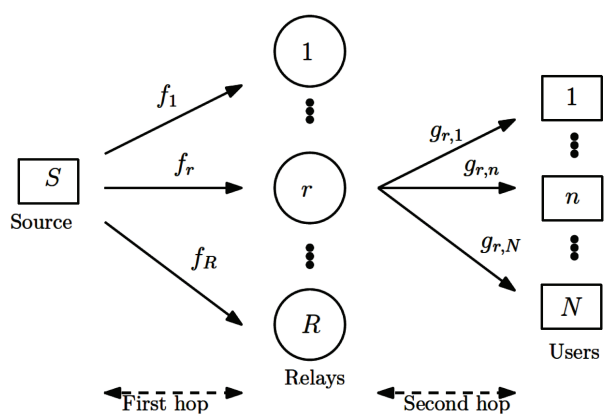

Fig. 1. Multicast network model with multiple relay nodes and users.

To achieve this, all relays feed back their minimum equivalent (source-relay-user) signal-to-noise ratio (SNR) to the source. This results in significant transmission delays (especially for a large number of relays) since more resources have to be allocated for feedback traffic rather than data transmission.

In this paper, we propose a relay selection algorithm that minimizes the feedback air-time under the assumption of noisy feedback channels. To the best of our knowledge, this is the first paper that studies feedback reduction and throughput scaling for relay-aided multicast networks. We exploit the theory of Compressive Sensing (CS) to reduce the feedback air-time of opportunistic relay-aided multicast networks. In addition to this, we derive closed form expressions for the scaling laws of the asymptotic maximum equivalent SNR and achievable rate for decode and forward and amplify and forward relaying protocols.

The remainder of this paper is organized as follows. In Section II, we introduce the network model. In Section III, we discuss our proposed feedback algorithm and an important CS result relevant to our work. In Section IV, we evaluate the performance of the proposed selection algorithm. In Section $\mathrm{V}$, we provide some simulation results, and we conclude our work in Section VI

\section{Network Model}

The network we consider consists of one source, $R$ relays and $N \ll R$ users as shown in Fig. 1. All users are assumed to be equally important and the source uses only one relay to broadcast its packets to all group users. An outage is declared if one or more users can not decode the relayed 
packets. The channels from the source to the relays and from the relays to the users are modeled as independent Rayleigh fading channels. We assume a symmetric time-division-duplex (TDD) setup where the channel gains remain constant during the feedback. We denote by $f_{r}$ and $g_{r, n}$ the complex channels between the source and the $r$ th relay, and the $r$ th relay and the $n$th user respectively. $f_{r}$ and $g_{r, n}$ are assumed to be independent and identically distributed (i.i.d.) zero-mean and unit-variance complex Gaussian random variables. All relays are assumed to by synchronized and additive white Gaussian noise with zero mean and variance $\sigma^{2}$ is assumed at each receiver.

\section{A. Cooperation Model}

We assume that the channels from the source to all users are weak enough so that all transmissions form the source to the users are neglected and transmission must take place in a two-hop fashion. To achieve this, the source selects a relay that maximizes the receive SNR for the worst user. To select the "best" relay, all relays estimate their equivalent SNRs with the source and the $N$ users, and then feed back their minimum equivalent SNR to the source. Following that, the source selects the relay with the largest equivalent SNR to broadcast its packets to the group users. We elaborate on those two steps in the sequel.

1) SNR Estimation: Regardless of the relaying technique (DF or AF), all relays estimate their first and second hop channels. Prior feedback, the source and users broadcast orthogonal pilots to all relays. The estimation error analysis is beyond the scope of this paper, and thus, we assume perfect SNR estimation at the relay side. Having the two hop channels, the $r$ th relay calculates its two hop equivalent SNR $\gamma_{r}^{e}$, which depends on the relaying technique (DF or AF), as follows [9]

$$
\gamma_{r}^{e}=\left\{\begin{array}{c}
\min \left(\gamma_{r}, \min _{n \in\{1, \ldots, N\}}\left(\gamma_{r, n}\right)\right), \text { for DF protocol } \\
\min \\
\frac{\gamma_{r} \gamma_{r, n}}{\gamma_{r}+\gamma_{r, n}+1}, \text { for AF protocol }
\end{array}\right.
$$

where $\gamma_{r}=\frac{P_{s}}{\sigma^{2}}\left|f_{r}\right|^{2}$ is the instantaneous source-relay SNR of the $r$ th relay and $\gamma_{r, n}=\frac{P_{r}}{\sigma^{2}}\left|g_{r, n}\right|^{2}$ is the instantaneous SNR between the $r$ th relay and the $n$th user. $P_{s}$ is the maximum source transmission power and $P_{r}$ is the $r$ th relay maximum transmission power. The following lemma characterizes the CDF of the equivalent SNR. by

Lemma 1: The CDF of the equivalent SNR in (1) is given

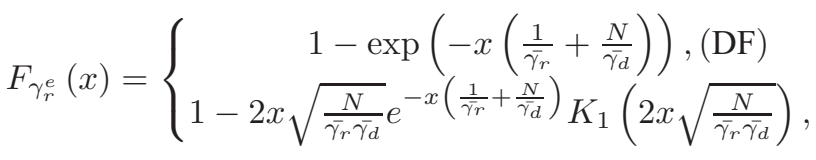

where $\overline{\gamma_{r}}$ and $\overline{\gamma_{d}}$ represent the average signal to noise ratio at the relays and the users respectively, and $K_{1}($.$) denotes the$ first order modified Bessel function of the second kind.

Proof: See Appendix A for proof.

\section{B. SNR Feedback Phase}

After estimating their equivalent SNRs, only strong relays will be able to access the shared feedback channel which is assumed to be slotted (into $M$ mini-slots). During each feedback channel (mini-slot), strong relays simultaneously feed back to the source. Each relay normalizes its transmission by its source channel, i.e. scales its transmission by $\frac{1}{f_{r}}$, and combines its equivalent SNR with its allocated feedback code and transmits the combination to the source. Let $\mathbf{v} \in \mathbb{R}^{R \times 1}$ be the vector representing the relays' equivalent SNR vector, $\mathbf{B}=\left[\begin{array}{llll}\mathbf{b}_{1} & \mathbf{b}_{2} & \ldots & \mathbf{b}_{R}\end{array}\right]$, where the vector $\mathbf{b}_{r} \in \mathbb{R}^{M \times 1}$, represent the relays' feedback code matrix, and the vector $\mathbf{y}$ represent the signal received at the source. We can then write the received vector $\mathbf{y}$ as follows

$$
\mathbf{y}=\mathbf{B v}+\mathbf{w}
$$

where the vector $\mathbf{w} \in \mathbb{R}^{M \times 1}$ represents the additive noise at the source. From $y$, the source obtains an estimate of the SNR vector $\mathbf{v}$, using CS theory as shown in the following section, and selects the relay with the highest equivalent SNR to broadcast its packets to the users.

\section{Opportunistic RELAy SELECTION}

\section{A. Compressive Sensing}

The concept of "sensing" a sparse signal through multiplication by an appropriate random matrix has been termed compressive sensing, and has recently gained popularity in many applications [10]-[16]. CS theory permits efficient reconstruction of the sensed signal with only a few sensing measurements. While there are many different methods used to solve sparse approximation problems (see e.g., [10]-[13]), we employ the least absolute shrinkage and selection operator (LASSO) as a recovery method as outlined in [11]. The choice of LASSO is due to the availability of a closed-form expression for the probability of sparsity pattern detection in the presence of noise. This probability term is essential for analyzing the performance of the proposed selection algorithm. Now, consider the following linear model

$$
\boldsymbol{y}=\mathrm{Bv}+\boldsymbol{w}
$$

where $\mathbf{B}$ is a real $M \times R$ sensing matrix, $\mathbf{v}$ is a real $R \times 1$ sparse vector ${ }^{1}$, and $\omega$ is a real $M \times 1$ vector of independent stochastic errors with zero mean Gaussian entries and variance $\sigma^{2}$. The LASSO estimate is defined as the solution to

$$
\arg \min _{\boldsymbol{\nu} \in \mathbb{R}^{R \times 1}} \frac{1}{2}\|\boldsymbol{y}-\mathbf{B} \boldsymbol{\nu}\|_{l_{2}}^{2}+\delta \sigma\|\boldsymbol{\nu}\|_{l_{1}}
$$

where $\|\cdot\|_{l_{1}}$ and $\|\cdot\|_{l_{2}}$ represents $l_{1}$ and $l_{2}$ norms, respectively, and $\delta$ is a regularization parameter. Given the following assumptions: (i) The matrix $\mathbf{B}$ is a real Gaussian matrix with unit normed columns and i.i.d. entries, (ii) $\min _{r \in \mathcal{S}}\left|v_{r}\right|>$ $8 \sigma \sqrt{2 \log R}$, where $\mathcal{S}=\left\{r: v_{r} \neq 0\right\}$ is the support of $\mathbf{v}$, and (iii) $\delta=2 \sqrt{2 \log R}$, then for relatively large $R$, and $M>C S \log R$, where $C$ is a positive constant (set as $C=2$ throughout this paper), the LASSO estimate identifies all nonzero entries of $\mathbf{v}$ with probability [11]

$$
\mathcal{P}_{\mathrm{cs}}(S) \geq 1-2 R^{-1}\left(\frac{1}{\sqrt{2 \pi \log R}}+\frac{S}{R}\right) \text {. }
$$

\footnotetext{
${ }^{1}$ A sparse vector is defined as a vector (of length $R$ ) that contains $S$ nonzero entries such that $S \ll R$.
} 


\section{B. Proposed Relay Selection Algorithm}

The goal of the proposed selection algorithm is to select the relay with the highest equivalent SNR and estimate its fed back SNR with minimal feedback air-time. In what follows, we provide a detailed discussion on the different stages of the proposed relay selection algorithm.

1) Relay ID Estimation: Instead of allocating each relay a dedicated feedback mini-slot, all relays share a pool of $M \ll R$ mini-slots for feedback. Each relay is allocated a Gaussian codeword of length $M$ for use during the feedback phase. The Gaussian codewords are perfectly known at the source and are drawn from the columns of a normalized real Gaussian matrix with zero mean and i.i.d. entries of variance $\frac{1}{M}$. To be able to apply the theory of CS, the fed back SNR vector should be sparse. To satisfy this condition, feedback is requested from only a few relays with an equivalent SNR higher than a threshold $\zeta$. As a result, only a few relays will feed back. The choice of threshold is made to yield a small outage probability $\mathcal{P}_{0}$. Using the CDF of the equivalent SNR given in (2), we can easily show that $\mathcal{P}_{o}=\left[F_{\gamma}(\zeta)\right]^{R}$. Therefore, the threshold $\zeta$ can be calculated as follows

$$
\zeta=F_{\gamma^{e}}^{-1}\left(\mathcal{P}_{0}^{1 / R}\right),
$$

where $F_{\gamma^{e}}^{-1}($.$) is the inverse CDF of the equivalent SNR.$ Once the source receives feedback from all strong relays, it applies the LASSO (see (5)) and estimates the IDs of the relays that fed back. If no relay is detected, a scheduling outage is declared. If at least one relay is detected, the source performs a subsequent SNR estimation and refinement steps as discussed below.

2) Equivalent SNR Estimation: Once the source estimates the sparsity pattern of $\mathbf{v}$ (or the identity of the strong relays), it prunes the columns of $\mathbf{B}$ that are associated to the inactive relays to obtain $\mathbf{B}_{S} \in \mathbb{R}^{M \times S}$. Hence, the received vector $\mathbf{y}$ in (3) can now be written as

$$
\mathbf{y}=\mathbf{B}_{S} \mathbf{v}_{S}+\mathbf{w}
$$

where $\mathbf{v}_{S}$ is obtained from the vector $\mathbf{v}$ by removing the entries associated with the inactive relays. Since $M>S$, the source can easily estimate the entries of $\mathbf{v}$. In particular, one can write the linear minimum mean square error estimate (LMMSE) after CS recovery as [18]

$$
\mathbf{v}_{S}^{\mathrm{LMMSE}}=\mathbf{B}_{S}^{T}\left(\mathbf{B}_{S} \mathbf{B}_{S}^{T}+\frac{\sigma^{2}}{\sigma_{v}^{2}} \mathbf{I}\right)^{-1} \mathbf{y}=\mathbf{v}_{S}+\mathbf{e}
$$

where the superscript ${ }^{T}$ stands for the transpose operation, $\sigma_{v}^{2}=\left|v_{r}\right|_{r \in \mathcal{S}}^{2}$ is the expected $r$ th relay feedback power, and $\sigma^{2}$ is the noise variance at the source. Here, the entries of the output noise vector, e, are Gaussian as linear operations preserve the Gaussian noise distribution ${ }^{2}$. Following [14], we can show that the variance of $\mathbf{e}$ is given by

- $\quad$ For $S=1$,

$$
\sigma_{e}^{2} \leq \sigma_{v}^{2} \frac{\rho^{-M / 2} e^{1 / 2 \rho}}{2^{M / 2}} \Gamma(1-M / 2,1 / 2 \rho),
$$

\footnotetext{
${ }^{2}$ While the elements of e are not necessarily independent, we will not use this fact in our back-off calculations (see Section III-C). Therefore, the backoff analysis here is pessimistic. This will result in a larger back-off and a small hit in the achievable throughput.
}

where $\rho=\frac{\sigma_{v}^{2}}{\sigma^{2}}$, and $\Gamma(.,$.$) is the incomplete gamma$ function.

- $\quad$ For $S=2$, the upper bound is shown in equation (11).

- When $S$ and $M$ tend to infinity such that $S / M \rightarrow$ $\beta \in[0,1]$,

$$
\sigma_{e}^{2} \leq \frac{\sigma_{v}^{2}}{1+\rho M(1-\sqrt{\beta})^{2}}
$$

\section{SNR Back-Off}

Due to the presence of additive noise in the feedback links, the estimated equivalent SNR in (9) might be higher or lower than the actual one. An estimated SNR larger than the actual SNR is problematic as it results in a transmission rate larger than the rate that can be supported by the minimum SNR user. Therefore, due to noisy feedback links, there is a need to back off the noisy recovered SNRs based on the noise variance. For each entry of the SNR vector in (3), a representative scalar equation takes the form $\mathrm{SNR}^{\prime}=\mathrm{SNR}+e$, where $\mathrm{SNR}$ and $\mathrm{SNR}^{\prime}$ stand for the actual and noisy SNRs, respectively, and $e$ represents the Gaussian noise. Now, if we consider a back-off on the received SNR by an amount $\Delta$, i.e. $\mathrm{SNR}^{\prime}=\mathrm{SNR}+e-$ $\Delta$, then, the back-off efficiency $\eta$, i.e. the probability that this backed off SNR is less than or equal to the actual fed back SNR is given by $\eta=\operatorname{Pr}\left[\mathrm{SNR}^{\prime} \leq \mathrm{SNR}\right]=\operatorname{Pr}[e \leq \Delta]=1-Q\left(\frac{\Delta}{\sigma_{e}}\right)$, where $\sigma_{e}$ is given by (10)-(12) and a proper choice of $\Delta$ is discussed in Section IV-B.

\section{Performance Analysis}

For the performance analysis, we consider the following metrics: 1) Feedback air-time, 2) Achievable rate, and 3) Achievable throughput.

\section{A. Feedback air-time}

The feedback air-time $L$, is defined as the average number of feedback mini-slots required to make a selection decision. In the proposed algorithm, $N+1$ mini-slots are required for all relays to estimate their channels with the source and the $N \ll R$ users, and $M$ mini-slots are required by the source to select the cooperating relay. Therefore, the total feedback air-time is

$$
L=M+N+1>2 \bar{S} \log R+N+1,
$$

where $\bar{S}=\mathrm{E}(S)$ is the average number of relays that report an equivalent SNR above the broadcasted threshold. The value of $\bar{S}$ is determined by the broadcasted threshold which is a function of $\mathcal{P}_{0}$ (see (7)).

\section{B. Achievable Rate}

In this section, we evaluate the rate achieved by the proposed selection algorithm for both $\mathrm{DF}$ and $\mathrm{AF}$ cases. The achievable rate $T$ depends on: (i) the back-off efficiency, (ii) the scheduling outage probability, and (iii) the success probability of the LASSO. Therefore, $T$ is derived as follows

$$
\begin{aligned}
T & \geq \frac{1}{2}\left(\log _{2}(1+\gamma-\Delta)\right)\left(1-Q\left(\frac{\Delta}{\sigma_{e}}\right)\right)\left(1-\mathcal{P}_{o}\right) \\
& \times\left(1-2 R^{-1}\left(\frac{1}{\sqrt{2 \pi \log R}}+\frac{\bar{S}}{R}\right)\right),
\end{aligned}
$$




$$
\sigma_{e}^{2} \leq \frac{\sigma_{v}^{2}}{(M-1) !(M-2) !}\left(\int_{0}^{\infty} \frac{\lambda^{M-2, \lambda} e^{-\lambda}}{1+\rho \lambda} \Gamma(M+1) d \lambda-2 \int_{0}^{\infty} \frac{\lambda^{M-1} e^{-\lambda}}{1+\rho \lambda} \Gamma(M, \lambda) d \lambda+\int_{0}^{\infty} \frac{\lambda^{M} e^{-\lambda}}{1+\rho \lambda} \Gamma(M-1, \lambda) d \lambda\right)
$$

where the inequality is due to the probability of CS detection shown in (6), the multiplication by $\frac{1}{2}$ is due to the half duplex operation, and $\gamma=\max _{r=1,2, \ldots, R}\left(\gamma_{r}^{e}\right)$ is the maximum equivalent SNR, which we will give its scaling law for the DF and $\mathrm{AF}$ cases in the following lemma.

Lemma 2: Let $\mu=\frac{1}{\overline{\gamma_{r}}}+\frac{N}{\overline{\gamma_{d}}}$ and $v=2 \sqrt{\frac{N}{\overline{\gamma_{r}} \overline{\gamma_{d}}}}$. Then as $R \rightarrow \infty$, the maximum equivalent $\operatorname{SNR}^{\gamma}=$ $\max _{r=1,2, \ldots, R}\left(\gamma_{r}^{e}\right)$ for DF and AF respectively scales as

$$
\gamma=\left\{\begin{array}{l}
\mu^{-1}(\xi+\log R), \text { DF relaying } \\
\left(\xi+\log \sqrt{\frac{\pi v(\mu+v)^{-1}}{2}}+\log R+\log \sqrt{\log R}\right) \\
\times(\mu+v)^{-1}, \text { AF relaying }
\end{array}\right.
$$

where $\xi=0.577$ is the Euler constant.

\section{Proof: See Appendix B for proof.}

In Fig. 2, we plot the mean of the maximum equivalent SNR $\gamma$ for different values of the average SNR per hop. As shown in the figure, for large $R\left(R=10^{4}\right)$, the numerical mean of the maximum equivalent SNR for both the DF and AF cases matches the theoretical results derived in (15).

It now remains to find an optimal value of $\Delta$ that maximizes the rate in (14). To achieve this, we differentiate $T$ in (14) with respect to $\Delta$ and set the resulted derivation to zero. This yields

$$
\left(\frac{1+\gamma-\Delta}{\sqrt{2 \pi} \sigma_{e}}\right) \exp \left(-\frac{\Delta^{2}}{2 \sigma_{e}^{2}}\right) \log (1+\gamma-\Delta)+Q\left(\frac{\Delta}{\sigma_{e}}\right)=1
$$

Solving this equation numerically, one can find the optimum $\Delta$ as a function of $\sigma_{e}$.

\section{Achievable Throughput}

In Section IV-B, we assumed the amount of air-time consumed during feedback transmission to be negligible. This is not generally a realistic assumption. To take into account the feedback air-time we define the throughput as the total achievable rate per feedback mini-slot. Using (14), the average throughput of the proposed algorithm is

$$
U=T / L,
$$

where $T$ and $L$ are derived in (14) and (13) respectively.

\section{Simulation Results}

In this section, we simulate the proposed selection algorithm under the assumption of a noisy feedback channel. For demonstration purposes, we only study the performance of the proposed algorithm in a network employing multiple DF relays. The proposed algorithm can be easily applied to a network with multiple AF relays. We compare the proposed algorithm with the noiseless dedicated feedback algorithm

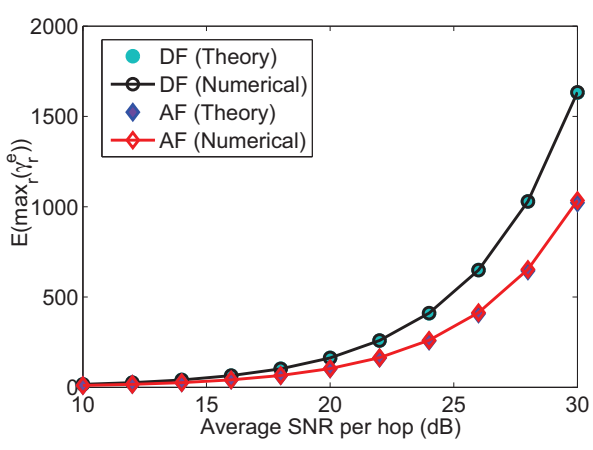

Fig. 2. Theoretical and numerical values for the expected value of the maximum equivalent SNR versus the average SNR per hop. $R=10^{4}$ relays, $\overline{\gamma_{r}}=\overline{\gamma_{d}}$, and $N=5$ users.

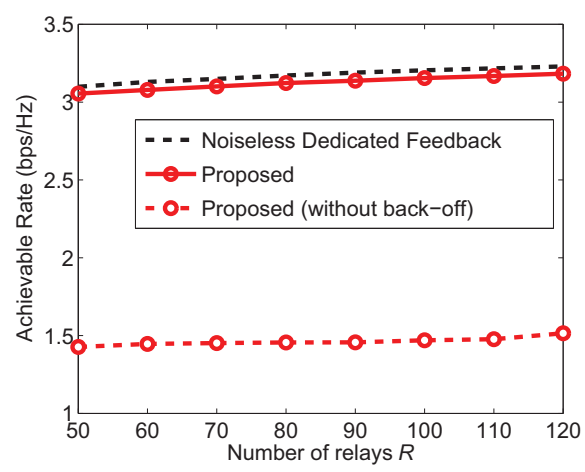

Fig. 3. Achievable rate versus the number of relays $R . \bar{S}=5, \overline{\gamma_{r}}=\overline{\gamma_{d}}=20$ $\mathrm{dB}$, and $N=5$ users.

where all the relays feed back their equivalent SNR in independent mini-slots. Unless otherwise specified, we assume $M=2.3 \bar{S} \log R$ in all simulations.

In Fig. 3, we plot the rate achieved (with and without SNR back-off) by the proposed algorithm for different values of $R$. As shown in Fig. 3, the rate (with LMMSE refinement and optimum SNR back-off) achieved by the proposed algorithm approaches the rate achieved by the noiseless dedicated feedback algorithm for all values of $R$. When no back-off is performed, the figure shows a hit in the achievable rate. The reason for this is that noise at the source might result in an estimated SNR larger than the equivalent SNR of the worst user. In that case, an outage is declared. The proposed algorithm optimally backs off to minimize over estimation errors.

In Fig 4, we plot the feedback air-time (in mini-slots) for different values of $R$. For the noiseless dedicated algorithm, the feedback grows linearly with the number of relays $R$, whereas, in the proposed algorithm, the feedback air-time grows logarithmically with $R$. As the feedback air-time increases, the throughput decreases as shown in Fig. 5, and as a result, the 


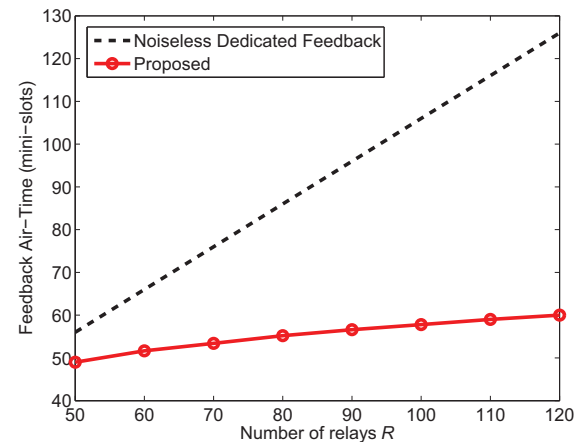

Fig. 4. Feedback air-time versus the number of relays $R . \bar{S}=5, \overline{\gamma_{r}}=$ $\overline{\gamma_{d}}=20 \mathrm{~dB}$, and $N=5$ users.

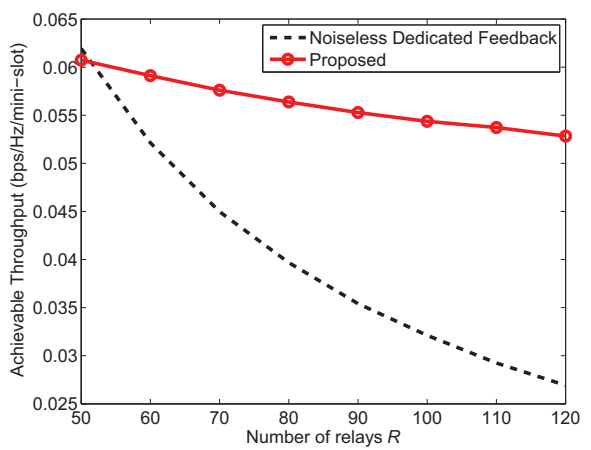

Fig. 5. Achievable throughput versus the number of relays $R . \bar{S}=5$, $\overline{\gamma_{r}}=\overline{\gamma_{d}}=20 \mathrm{~dB}$, and $N=5$ users.

proposed algorithm out-performs the noiseless dedicated feedback algorithm. The achievable throughput for the noiseless dedicated feedback algorithm decreases dramatically with $R$ when compared to our proposed algorithm. This is due to the high feedback air-time requirement of the dedicated feedback algorithm when compared to the proposed feedback algorithm.

\section{CONCLUSION}

In this paper, we introduced a generic CS-based feedback algorithm for relay selection in a multicast network. The proposed algorithm, with noisy feedback estimates, is shown to reduce the feedback air-time required to select a broadcasting relay and achieve a rate that compares favorably with ideal noiseless dedicated feedback algorithms. For large number of relays, the throughput achieved by the proposed algorithm is shown to outperform the throughput achieved by dedicated feedback algorithms. Using extreme value theory, we derived the scaling laws of the equivalent SNR at the receiver for both DF and AF cases. We also showed that noise in the feedback links introduces a hit in the achievable rate. To minimize the effect of this noise, we introduced a back-off strategy that optimally backs off the noisy estimated SNRs.

\section{APPENDIX A}

\section{DERIVATION OF THE CDF OF THE EQUIVALENT SNR}

In this Appendix, we proof the CDF of the equivalent SNR given in (2). For the DF case, we can see from (1) that the equivalent SNR is the minimum between two exponential random variables $\gamma_{r}$ and $\gamma_{2}=\min _{n \in\{1, \ldots, N\}} \gamma_{r, n}$ with mean $\bar{\gamma}_{r}$ and $\frac{\overline{\gamma_{d}}}{N}$ respectively. Therefore,

$$
F_{\gamma_{r}^{e}}(x)=1-\operatorname{Pr}\left(\gamma_{r} \geq x\right) \operatorname{Pr}\left(\gamma_{2} \geq x\right)=1-e^{-x\left(\frac{1}{\gamma_{r}}+\frac{N}{\gamma_{d}}\right)} .
$$

For the AF case, as shown in (1), the $N$ SNRs are not necessarily independent, therefore, to determine the CDF of the equivalent SNR, we need to condition on $\gamma_{r}$ and then integrate over its range. Assuming high SNR, $\frac{\gamma_{r} \gamma_{r, n}}{\gamma_{r}+\gamma_{r, n}+1} \approx \frac{\gamma_{r} \gamma_{r, n}}{\gamma_{r}+\gamma_{r, n}}$ [9]. Hence,

$$
\begin{array}{r}
\operatorname{Pr}\left(\gamma_{r}^{e} \leq x \mid \gamma_{r}\right)=1-\prod_{n=1}^{N} \operatorname{Pr}\left(\frac{\gamma_{r} \gamma_{r, n}}{\gamma_{r}+\gamma_{r, n}} \geq x \mid \gamma_{r}\right) \\
=1-\operatorname{Pr}\left(\frac{\gamma_{r} \gamma_{r, n}}{\gamma_{r}+\gamma_{r, n}} \geq x \mid \gamma_{r}\right)^{N},
\end{array}
$$

since all the relay-destination SNRs are identically distributed. Integrating over all range of $\gamma_{r}$, we have

$$
\begin{aligned}
F_{\gamma_{r}^{e}}(x) & =1-\int_{0}^{\infty} \operatorname{Pr}\left(\frac{\gamma_{r} \gamma_{r, n}}{\gamma_{r}+\gamma_{r, n}} \geq x \mid \gamma_{r}=t\right)^{N} f_{\gamma_{r}}(t) d t \\
& =1-\int_{x}^{\infty} \operatorname{Pr}\left(\gamma_{r, n} \geq \frac{t x}{t-x}\right)^{N} \frac{1}{\overline{\gamma_{r}}} \exp \left(-\frac{t}{\overline{\gamma_{r}}}\right) d t \\
& =1-\int_{x}^{\infty} \frac{1}{\overline{\gamma_{r}}} \exp \left(-\frac{t}{\overline{\gamma_{r}}}-\frac{N t x}{\overline{\gamma_{d}}(t-x)}\right) d t
\end{aligned}
$$

With the change of variables, $\theta=t-x$, (18) becomes

$$
\begin{aligned}
F_{\gamma_{r}^{e}}(x) & =1-\int_{0}^{\infty} \frac{1}{\overline{\gamma_{r}}} \exp \left(-\frac{\theta+x}{\overline{\gamma_{r}}}-\frac{N(\theta+x) x}{\overline{\gamma_{d}} \theta}\right) d \theta \\
& =1-e^{-x\left(\frac{1}{\gamma_{r}}+\frac{N}{\bar{\gamma}_{d}}\right)} \int_{0}^{\infty} \frac{1}{\overline{\gamma_{r}}} \exp \left(-\frac{\theta}{\overline{\gamma_{r}}}-\frac{4 N x^{2}}{4 \overline{\gamma_{d}} \theta}\right) d \theta
\end{aligned}
$$

Using [17] (p. 337), we get

$$
F_{\gamma_{r}^{e}}(x)=1-2 x \sqrt{\frac{N}{\overline{\gamma_{r}} \overline{\gamma_{d}}}} e^{-x\left(\frac{1}{\gamma_{r}}+\frac{N}{\bar{\gamma}_{d}}\right)} K_{1}\left(2 x \sqrt{\frac{N}{\overline{\gamma_{r}} \overline{\gamma_{d}}}}\right) .
$$

This completes the proof of the lemma.

\section{APPENDIX B}

\section{LIMIT BEHAVIOR OF MAXIMA}

In this section, using extreme value theory, we introduce three extreme value distributions that are useful for the proof of the Lemma 2. Consider the stochastic behavior of the maximum $X_{R}^{*}=\max \left(x_{1}, x_{2}, \ldots, x_{R}\right)$ of $R$ identically distributed random variables $x_{1}, x_{2}, \ldots, x_{R}$ with $\mathrm{CDF} F(x)$. The CDF of $X_{R}^{*}$ is

$$
\begin{aligned}
F\left(X_{R}^{*}\right)=\operatorname{Pr}\left(X_{R}^{*} \leq x\right) & =\operatorname{Pr}\left(X_{1} \leq x, \ldots, X_{R} \leq x,\right) \\
& =\prod_{r=1}^{R} \operatorname{Pr}\left(X_{1} \leq x\right)=[F(x)]^{R}
\end{aligned}
$$

Now, if there exits constants $a_{R}$ and $b_{R}$ such that $\operatorname{Pr}\left(\frac{X_{R}^{*}-b_{R}}{a_{R}} \leq x\right)=\left[F\left(a_{R} x+b_{R}\right)\right]^{R} \rightarrow G(x)$ as $R \rightarrow \infty$ at all continuity points of $G(x)$, then $G(x)$ falls into one of 
the following distributions [19]:

(i) (Frechet) $G_{1}(x ; \alpha)=e^{-x^{-\alpha}} u(x), \alpha>0$

(ii) (Weibull) $G_{2}(x ; \alpha)=e^{-(-x)^{\alpha}} u(-x), \alpha>0$

(iii) (Gumbel) $G_{3}(x)=e^{-e^{-x}}, \alpha>0$,

where $u($.$) is the step function. To find the scaling law of \gamma$ in Section IV-B, we need to find sequences $a_{R}$ and $b_{R}$ such that $F_{\gamma}\left(a_{R} x+b_{R}\right) \rightarrow G(x)$ as $R \rightarrow \infty$ at all continuity points of $G(x)$. For the DF case, if we let $a_{R}=\left(\frac{\bar{\gamma}_{r} \bar{\gamma}_{d}}{N \bar{\gamma}_{r}+\bar{\gamma}_{d}}\right)$, and $b_{R}=\left(\frac{\bar{\gamma}_{r} \bar{\gamma}_{d}}{N \bar{\gamma}_{r}+\bar{\gamma}_{d}}\right) \log R$, then we have

$$
\begin{aligned}
F_{\gamma}\left(a_{R} x+b_{R}\right) & =\left[1-e^{-\left(a_{R} x+b_{R}\right)\left(\frac{1}{\gamma_{r}}+\frac{N}{\gamma_{d}}\right)}\right]^{R} \\
& =\left(1-\frac{1}{R} e^{-x}\right)^{R} \rightarrow G_{3}(x)=e^{-e^{-x}},
\end{aligned}
$$

which is the Gumbel distribution. Evidently, its mean is $E\left[a_{R} x+b_{R}\right]=a_{R} \xi+b_{R}$. Therefore, as $R \rightarrow \infty$, the expected value of the maximum equivalent SNR achieved by $R$ i.i.d. relays operating under $\mathrm{DF}$ protocol scales as $\gamma \rightarrow\left(\frac{\bar{\gamma}_{r} \bar{\gamma}_{d}}{N \bar{\gamma}_{r}+\bar{\gamma}_{d}}\right)(\xi+\log R)$. For AF relaying, recall that $K_{1}(x) \approx \sqrt{\frac{\pi}{2 x}} e^{-x}$ for high $x$ (or high SNR). Therefore, under high SNR assumption, the CDF of $\gamma_{r}^{e}$ can be approximated by (see (2))

$$
\begin{aligned}
F_{\gamma_{r}^{e}}(x) & \approx 1-2 x \sqrt{\frac{N}{\bar{\gamma}_{r} \bar{\gamma}_{d}}} e^{-x\left(\frac{1}{\bar{\gamma}_{r}}+\frac{1}{\bar{\gamma}_{d}}\right)} \sqrt{\frac{\pi}{4 x \sqrt{\frac{N}{\bar{\gamma}_{r} \bar{\gamma}_{d}}}} e^{-2 x \sqrt{\frac{N}{\bar{\gamma}_{r} \bar{\gamma}_{d}}}}} \\
& \approx 1-\sqrt{\frac{\pi v x}{2}} e^{-(\mu+v) x},
\end{aligned}
$$

where $v=2 \sqrt{\frac{N}{\bar{\gamma}_{r} \bar{\gamma}_{d}}}$ and $\mu=\left(\frac{1}{\bar{\gamma}_{r}}+\frac{N}{\bar{\gamma}_{d}}\right)$. Using (22), the CDF of $\gamma=\max \left(\gamma_{1}^{e}, \ldots, \gamma_{R}^{e}\right)$ can be expressed as follows

$$
F_{\gamma}(x)=\left[1-\sqrt{\frac{\pi v x}{2}} e^{-(\mu+v) x}\right]^{R} .
$$

To find the scaling law of $\gamma$, we need to find sequences $a_{R}$ and $b_{R}$ such that $F_{\gamma}\left(a_{R} x+b_{R}\right) \rightarrow G(x)$ as $R \rightarrow \infty$ at all continuity points of $G(x)$. By choosing $a_{R}=(\mu+v)^{-1}$ and $b_{R}=$ $(\mu+v)^{-1}\left(\log \sqrt{\frac{\pi v}{2}(\mu+v)^{-1}}+\log R+\log \sqrt{\log R}\right)$,

becomes

$$
\begin{aligned}
& {\left[F_{\gamma}\left(a_{R} x+b_{R}\right)\right]^{1 / R}=1-\left(\frac{\pi v}{2}(\mu+v)^{-1}\right)^{1 / 2}} \\
& \times\left(x+\log \sqrt{\frac{\pi v(\mu+v)^{-1}}{2}}+\log R+\log \sqrt{\log R}\right)^{1 / 2} \\
& \times e^{-x-\log \sqrt{\frac{\pi v(\mu+v)^{-1}}{2}}-\log R-\log \sqrt{\log R}} \\
& =1-\left(x+\log \sqrt{\frac{\pi v(\mu+v)^{-1}}{2}}+\log R+\log \sqrt{\log R}\right)^{1 / 2} \\
& \times \frac{1}{R} \frac{1}{\sqrt{\log R}} e^{-x} \\
& =1-\frac{1}{R} e^{-x} \sqrt{\frac{x}{\log R}+\frac{\log \sqrt{\frac{\pi v(\mu+v)^{-1}}{2}}}{\log R}+1+\frac{\log \sqrt{\log R}}{\log R} .}
\end{aligned}
$$

[7] A. Sendonaris, E. Erkip, and B. Aazhang, "User cooperation diversity. part i. system description," IEEE Trans. Commun., vol. 51, no. 11, pp. 1927-1938, Nov. 2003.

[8] Y. Jing, and H. Jafarkhani, "Single and multiple relay selection schemes and their achievable diversity orders," IEEE Trans. Wireless. Commun., vol. 8, no. 3, pp. 1414-1423, Mar. 2009.

[9] M. Hasna, and M.-S. Alouini, "End-to-end performance of transmission systems with relays over Rayleigh fading channels," IEEE Trans. Wireless Commun, vol. 2, no. 6, pp. 1126-1131, 2003.

[10] D. Donoho, "Compressed Sensing," IEEE Trans. Inf. Theory, vol. 52, no. 4, pp. 1289-1306, Apr. 2006.

[11] E. Candes and Y. Plan, "Near-Ideal Model Selection by $l_{1}$ Minimization," Ann. Statist., vol. 37, no. 5A, pp. 2145-2177, 2009.

[12] M. Masood, and T. Al-Naffouri, "Sparse Reconstruction Using Distribution Agnostic Bayesian Matching Pursuit", IEEE Trans. Signal Process., vol.61, no.21, pp.5298-5309, Nov. 1, 2013.

[13] R. Baraniuk, "Compressive Sensing", IEEE Signal Process. Mag., vol. 24 , no. 4, pp. 118-121, July 2007.

[14] M. Eltayeb, T. Al-Naffouri, and H. Bahrami, "Compressive Sensing for Feedback Reduction in MIMO Broadcast Channels," IEEE Trans. Commun., to appear, 2014.

[15] S. Qaseem, T. Al-Naffouri, and T. Al-Murad, "Compressive sensing based opportunistic protocol for exploiting multiuser diversity in wireless networks", in IEEE Int. Symposium on Personal, Indoor and Mobile Radio Commun., Tokyo, Japan, Sept. 2009.

[16] S. T. Qaseem and T. Y. Al-Naffouri, "Compressive sensing for feedback reduction in MIMO broadcast channels", in IEEE Int. Conf. on Commun. (ICC), Cape Town, South Africa, May 2010.

[17] I. Gradshteyn, and I. Ryzhik, Table of integrals, series, and products, 7th ed. Elsevier, 2007.

[18] A. Sayed, Fundamentals of Adaptive Filtering, John Wiley \& Sons, 2003.

[19] H. David, Order Statistics, New York, Wiley, 1970. 